\title{
BMJ Open Are interventions to improve cardiovascular disease risk factors in premenopausal women effective? A systematic review and meta-analysis
}

\author{
Lan Gao (D) , Jan Faller, Ishani Majmudar, Phuong Nguyen (D) , Marj Moodie
}

To cite: Gao L, Faller J, Majmudar I, et al. Are interventions to improve cardiovascular disease risk factors in premenopausal women effective? A systematic review and meta-analysis. BMJ Open 2021;11:e042103. doi:10.1136/ bmjopen-2020-042103

- Prepublication history and additional supplemental material for this paper are available online. To view these files, please visit the journal online (http://dx.doi.org/10.1136/ bmjopen-2020-042103).

Received 25 June 2020 Accepted 13 July 2021
Check for updates

(C) Author(s) (or their employer(s)) 2021. Re-use permitted under CC BY-NC. No commercial re-use. See rights and permissions. Published by BMJ.

Deakin Health Economics, Deakin University Faculty of Health, Burwood, Victoria, Australia

Correspondence to

Dr Lan Gao;

lan.gao@deakin.edu.au

\section{ABSTRACT}

Objectives Non-traditional risk factors place young women at increased risk of cardiovascular disease (CVD) over their lifetime. The current study undertakes a systematic review and meta-analysis of randomised controlled trials (RCTs) that examined the effectiveness of primary prevention interventions for CVD in premenopausal women.

Methods An electronic literature search was performed in key databases in July 2018 and updated in May 2020. RCTs that recruited predominately female participants with a proportion aged under 55 years and that compared primary prevention interventions of CVD with usual practice were included. Two reviewers undertook the selection process for study inclusion. Meta-analysis was conducted for studies based on the same intervention in order to synthesise the results.

Results 14 RCTs with sample size ranging from 49 to 39 876 were included. Interventions included diet (2), vitamin E/antioxidants (3), lifestyle modification programme (7) and aspirin (2). The meta-analysis results indicated that diet nor vitamin E/antioxidant did not significantly lower the CVD risk profiles, while lifestyle modification programme involving components of lifestyle education, counselling and multiple follow-ups showed great potential to improve risk profiles. The lifestyle modification intervention improved blood pressure $(-2.11 \mathrm{~mm} \mathrm{Hg}, 95 \% \mathrm{Cl}-4.32$ to 0.11 , for systolic and $-3.31 \mathrm{~mm} \mathrm{Hg}(95 \% \mathrm{Cl}-4.72$ to -1.91 , for diastolic), physical activity (30.72 MET-min/ week, $95 \%$ Cl 23.57 to 37.87 , for moderate physical activity $12.70 \mathrm{MET}$-min/week, 95\% Cl 8.27 to 17.14 , for vigorous physical activity) and fasting blood glucose $(-0.37 \mathrm{mmol} / \mathrm{L}, 95 \% \mathrm{Cl}-0.58$ to -0.15$)$. Subgroup metaanalysis in studies with a mean age under 51 years old suggested that lifestyle modification intervention remained to be effective in improving physical activity and fasting blood glucose.

Conclusion The effective interventions identified in this review although with a small sample size and short duration could potentially inform future design of primary prevention of CVD in premenopausal women.

\section{INTRODUCTION}

Cardiovascular disease (CVD) is one of the leading causes of death among women worldwide despite the substantial advances

\section{Strengths and limitations of this study}

The first systematic review for primary prevention of cardiovascular disease in young women.

- Results could guide the design of future clinical trials.

- Interventions involving lifestyle modifications were promising.

- None of the included studies specifically focused on premenopausal women.

- Small number of included studies did not permit accurate assessments of publication bias.

in disease awareness, prevention and treatment. ${ }^{1}$ Early detection and managing women at risk of CVD will prevent hospital admissions, save lives and improve the quality of life of those affected. Therefore, primary prevention of CVD is receiving extensive attention in the research field.

Primary prevention normally involves modification of CVD risk factors. Traditional CVD risk factors include obesity, hypertension, dyslipidaemia, diabetes, physical inactivity, excessive alcohol consumption and smoking, while non-traditional CVD risk factors comprise pregnancy-related disorders, such as gestational diabetes and hypertension, preterm delivery (PTD) and endocrine disorders in women of reproductive age (ie, polycystic ovary syndrome and early menopause). ${ }^{2-4}$ For example, premenopausal women without a history of PTD are relatively protected against CVD, whereas women with such a history are prone a significantly earlier onset of CVD, ${ }^{2}$ resulting in higher losses in terms of premature mortality and productivity. Meanwhile, other CVD risk factors, even though not exclusive to women, have much higher prevalence in women than men (ie, migraine which is associated with risk of stroke, occurs three times more often in women). ${ }^{56}$ The American Heart Association 
(AHA)/American Stroke Association guidelines for the prevention of CVD in women recommend CVD risk assessment in women with certain reproductive manifestations of CVD risk (such as pregnancy-related adverse outcomes) and suggest that female-specific risk factors may improve/complement the current CVD risk assessment strategies. ${ }^{78}$ To combat the ever increasing disease burden of CVD, identifying effective ways to prevent CVD in specific risk groups potentially offers the best solutions; this provoked the interest to uncover interventions that are tailored to altering the CVD risk profiles of premenopausal women.

While research around primary prevention of CVD has examined a wide range of interventions from pharmaceuticals to lifestyle modification programmes, these studies have generally recruited people of older age (ie, postmenopausal) and/or both gender groups. ${ }^{9-11}$ There is a growing appreciation that there may be gender differences in the magnitude of the relative and absolute potential benefits and risks associated with preventive interventions. ${ }^{12} 13$ Hence, evidence from studies in unrestricted populations is not necessarily applicable to women of younger age (ie, premenopausal). Besides, there is no specific study that has been designed to investigate how to improve the CVD risk profiles for women with histories of pregnancy-related complications (ie, PTD, gestational diabetes).

In light of (1) the clinical need to look for effective primary prevention interventions that can alter the trajectory of CVD development for women with nontraditional CVD risk factors identified (ie, PTD, diagnosis of polycystic ovary syndrome, etc) earlier in their lives and (2) the potential for gender-based and even age-based differences (ie, some interventions may be more effective in younger women) in CVD, ${ }^{14-16}$ studies are warranted to explore the primary prevention of CVD for women of reproductive age. An ideal way to address this research question would be to conduct a randomised controlled trial (RCT) to compare various interventions in this specific group of women, but is prohibitive in terms of resources and time required. Alternatively, we can capitalise on existing studies to provide timely, preliminary evidence that may assist with selecting an optimal intervention for further investigation given that there are at least two broad groups of primary prevention interventions, including medications (ie, aspirin, ${ }^{17}$ statins, ${ }^{13}$ vitamin $\mathrm{E},{ }^{18}$ etc), physical activity/counselling in lifestyle modification programmes ${ }^{19}$ for CVD in the general population.

The current study undertakes a systematic review of RCTs that examined the effectiveness of primary prevention interventions for CVD in premenopausal women and meta-analyses the effects for studies investigating the same type of intervention. The aim is to provide evidence to identify a candidate intervention that is likely to be effective in young women with non-traditional CVD risk factors (ie, pregnancy-related complications, endocrine disorders, migraine, etc).

\section{METHODS}

\section{Literature search}

An electronic literature search was conducted in Medline (plus PsycINFO vis EBSCO) and Embase. The search was carried out in July 2018 using the key search terms including women, primary prevention, CVD (myocardial infarction (MI), stroke and heart failure), with filter for RCTs. Studies that met the following criteria were included: (1) comprise a cohort of females (at least $50 \%$ of all participants were female) and with a mean age of 55 years or under with no established CVD (55 was selected as a crude proxy due to the absence of cut-off age for menopausal status) ${ }^{20-22}$; (2) participants randomised to intervention or usual care (or placebo) and (3) report at least one of the following outcomes: numbers of major adverse cardiovascular events (MI, stroke, coronary revascularisation, cardiac sudden death, angina), all-cause mortality, incident case of diabetes, surrogate outcome (blood pressure (BP), lipids, body mass index (BMI), level of physical activity, inflammatory markers); (4) publication is presented fully in English; (5) control group for these interventions received the standard care as per the current practice. Exclusion criteria were trials that included predominantly (ie, $>50 \%$ ) male participants and/or females age $>55$ years; trials with small samples (ie, recruited less than 30 participants); studies published prior to 2000 were excluded as the aim was to identify contemporary evidence to guide the design of future interventions. Studies meeting the inclusion criteria based on full-text examination were assessed and extracted for further analysis by two reviewers (JF and IM). Predesigned tables were used to guide the data extraction. Data on patient characteristics, study design, duration of follow-up, intervention characteristics and outcomes were extracted. The quality of the included studies was evaluated individually following the Cochrane Risk of Bias $\mathrm{Tool}^{23}$ with due consideration of six domains (ie, judgement on the risk of bias will be made for each domain based on three categories: high risk, low risk and unclear risk of bias). The detailed search strategies are provided in online supplemental file 1 .

\section{Data analysis}

Interventions were grouped for further analysis based on their nature of delivery/content of the intervention. Where more than two studies evaluated the same intervention, the outcomes were meta-analysed using inverse variance method with either a fixed or random effects model in Review Manager (V.5.3, The Cochrane Collaboration, 2014) depending on the magnitude of the heterogeneity across the studies ( $>50 \%$ suggests substantial heterogeneity). If the results from subgroup (ie, women aged younger than 55 years old) were reported, they were extracted for the analysis. Summary effects were reported as relative risk (RR) with corresponding 95\% CI if the outcome was dichotomous or as a mean difference (MD) with the $95 \% \mathrm{CI}$ if the outcome was continuous. Heterogeneity across RCTs were assessed using $\chi^{2}$ test and $\mathrm{I}^{2}$ 
statistic. Forest plots were used to report results of metaanalysis. Publication bias were assessed using funnel plots.

\section{Subgroup analysis}

Where applicable, subgroup analysis was performed to examine the intervention effect in subgroups of women with a younger cut-off age for menopausal status (ie, $<51$ years old) or varied characteristics, for example, baseline CVD risk factors.

The methods for systematic review and meta-analysis followed the Preferred Reporting Items for Systematic Reviews and Meta-Analyses statement.

\section{Patient and public involvement}

Patients or the public were not involved in the design, or conduct, or reporting, or dissemination plans of the systematic review.

\section{RESULTS}

\section{Literature search}

The initial literature search through Embase, Medline, CINAHL and Cochrane Library identified 1185, 471, 127 and 38 citations, respectively. The updated search in May 2020 identified an additional 424 citations. From the total 2245 retrieved citations, 505 were duplicates as identified by Endnote. A total of 1740 articles were screened for title and abstract, from which 1209 articles were excluded due to irrelevance $(n=864)$, duplicates found manually $(\mathrm{n}=151)$, articles published before $2000(\mathrm{n}=189)$ and not in English language $(n=5)$. The remaining 531 articles were assessed on a full-text basis; 517 were excluded due to non RCTs $(n=375)$, age older than 55 years $(n=81)$, majority being males $(n=1)$, sample size less than 30 $(n=3)$, prior histories of CVD $(n=2)$, duplicate publications of included studies $(n=2)$, irrelevant outcomes $(\mathrm{n}=6)$ and non-premenopausal studies $(\mathrm{n}=47)$. Thus, a total of $14^{171924-35}$ articles were included into this systematic review. The study selection process is illustrated in figure 1. Key characteristics of the included studies are summarised in table 1. Details of each study are supplied in online supplemental tables.

\section{Types of interventions}

Since the interventions in the included studies were heterogeneous and reported different outcomes that related to CVD primary prevention, the studies have been grouped by type of intervention for further analysis. There are four broad types of interventions for primary prevention of CVD in women:

1. Diet intervention, $\mathrm{N}=2$.

2. Lifestyle modification intervention (ie, modification of diet and physical activity), $\mathrm{N}=7$.

3. Vitamin $\mathrm{E} /$ antioxidant, $\mathrm{N}=3$.

4. Aspirin, $\mathrm{N}=2$.

Four studies included data from the Women's Health Study which was a large randomised, double-blind, placebo-controlled trial to examine the benefits and risk

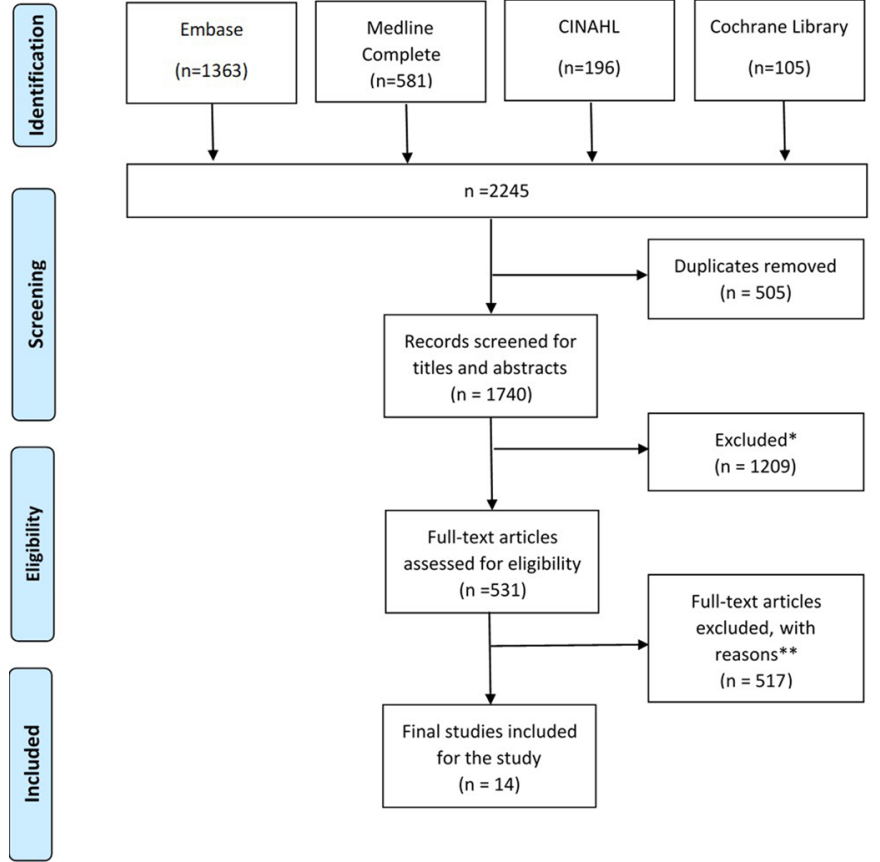

Figure 1 PRISMA flow diagram for the selection of included studies in the systematic review. *Reasons for exclusion: Manual removal of futher duplicates $(n=151)$. Not in English $(n=5)$. Title and abstract not relavant $(n=864)$, Older than 2000 $(n=189) .{ }^{*}$ Reasons for exclusion: Females $<55$ years of age $(n=81)$. Existing CVD $(n=2)$. Outcomes not relavant $(n=6)$. Part of an included articles $(n=2)$. Non-premenopausal females $(n=47)$. Majority men $(n=1)$. Sample size $<30(n=3)$. NonRCT study ( $n=375)$. CVD, cardiovascular disease; PRISMA, Preferred Reporting Items for Systematic Reviews and MetaAnalyses; RCT, randomised controlled trial.

of low dose aspirin and vitamin $\mathrm{E}$ in the primary prevention of CVD for women. ${ }^{1728} 2932$ Moore et al compared four interventions in a single study, which were treated as separate studies in the analysis. ${ }^{35}$

\section{Characteristics of the included studies}

Generally, the included RCTs were heterogeneous in terms of intervention (diet interventions, medications, lifestyle modifications), populations (women only or mixed-sex cohorts), age groups (premenopausal only or mixed premenopausal and postmenopausal), intervention duration (ranged from $2^{19}$ weeks to 10 years, ${ }^{28}$ primary/secondary outcomes (eg, incidence of first CVD events, changes in CVD risk factors and level of physical activities, urinary potassium excretion, etc), sample size (ranged from 49 to over 39,000), length of follow-up (from 2 weeks to 10 years) and setting (Asia, Africa, South America, Europe). The key characteristics of the included studies are summarised in table 1.

\section{Risk of bias assessment of included studies}

The majority of studies were rated as low risk of bias for selection (14/14), performance $(8 / 14)$, detection $(9 / 14)$, attrition $(11 / 14)$ and reporting (12/14) bias. Overall, two studies involving five publications 1728293234 were considered low risk of bias in all assessed domains; 


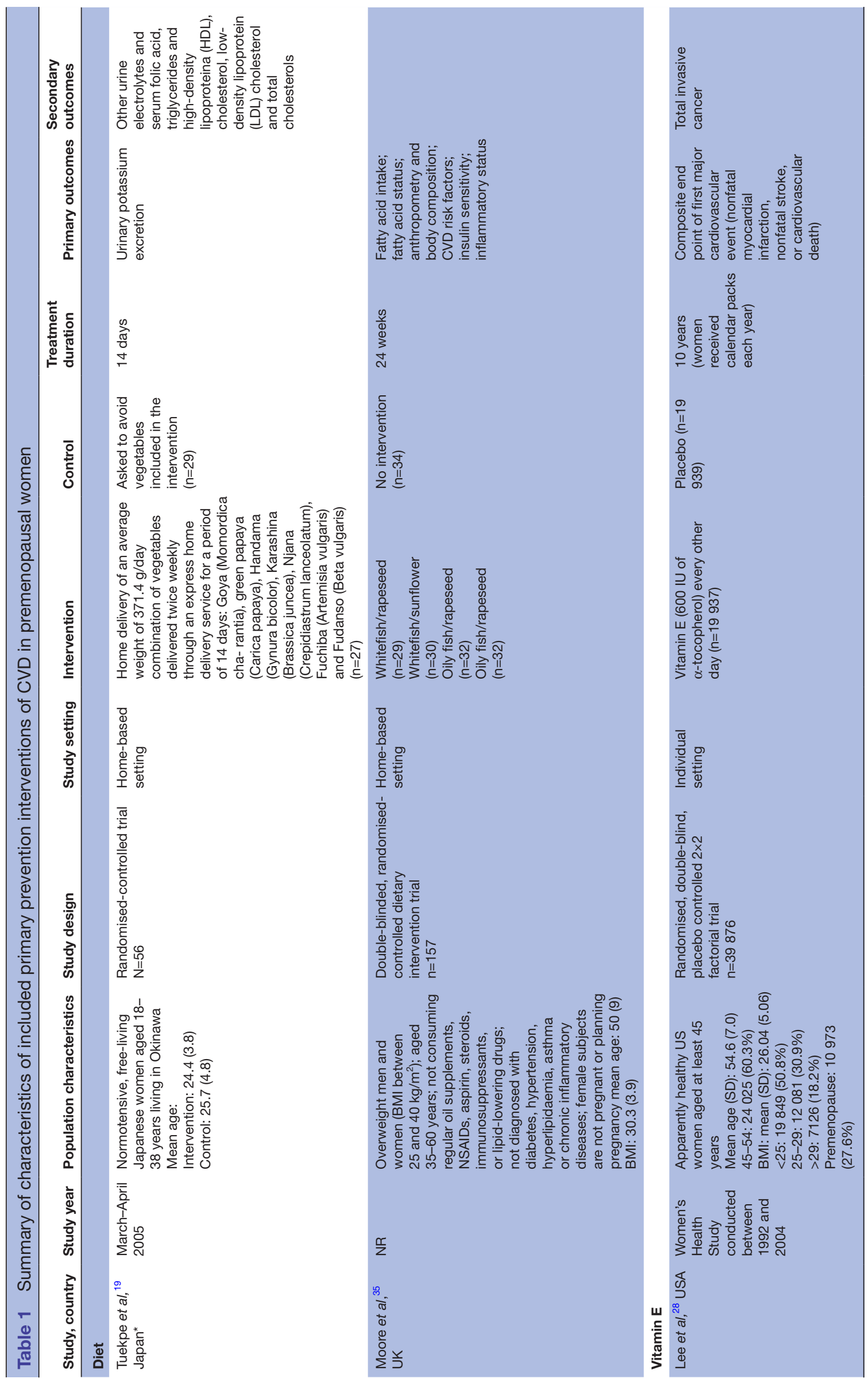




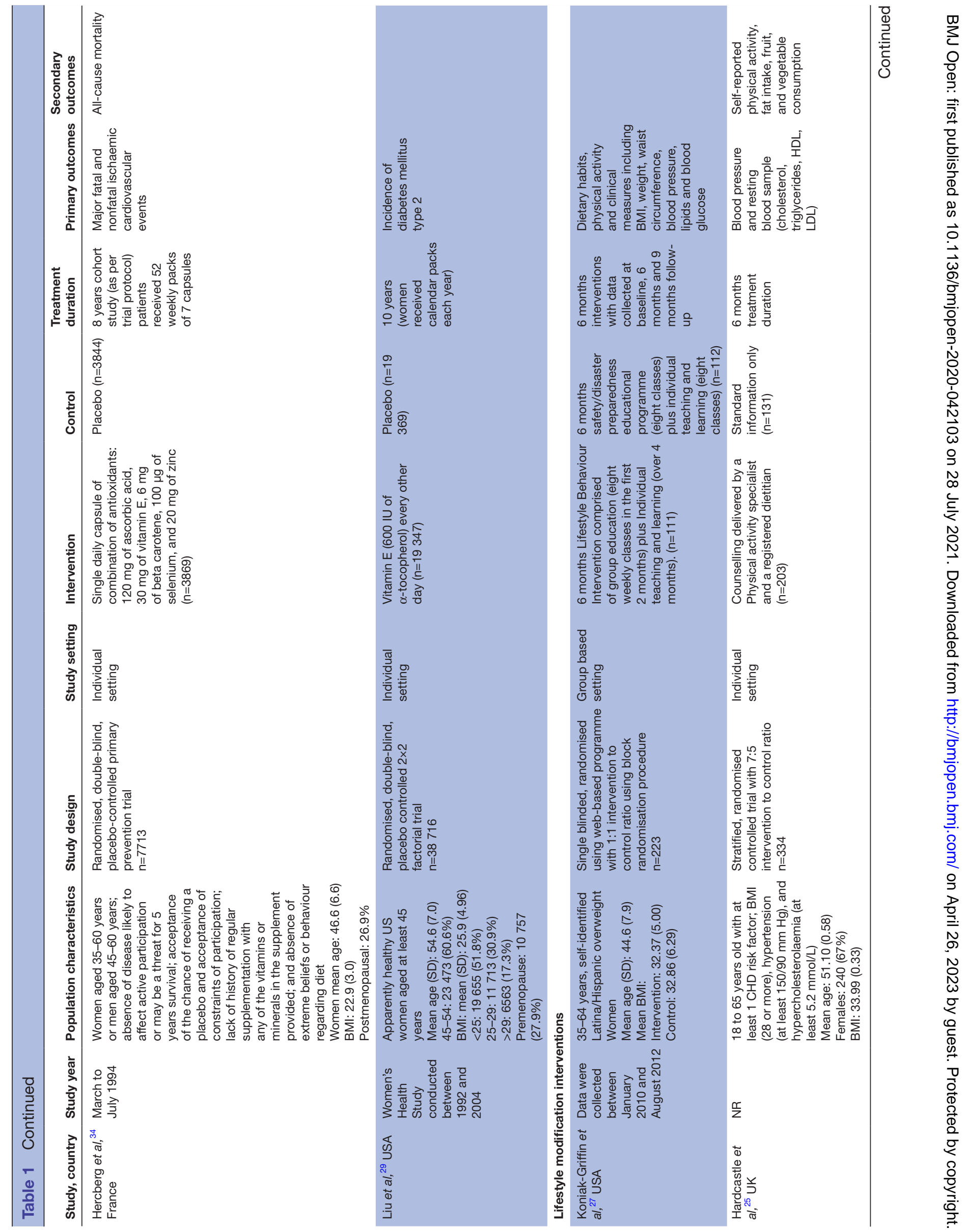



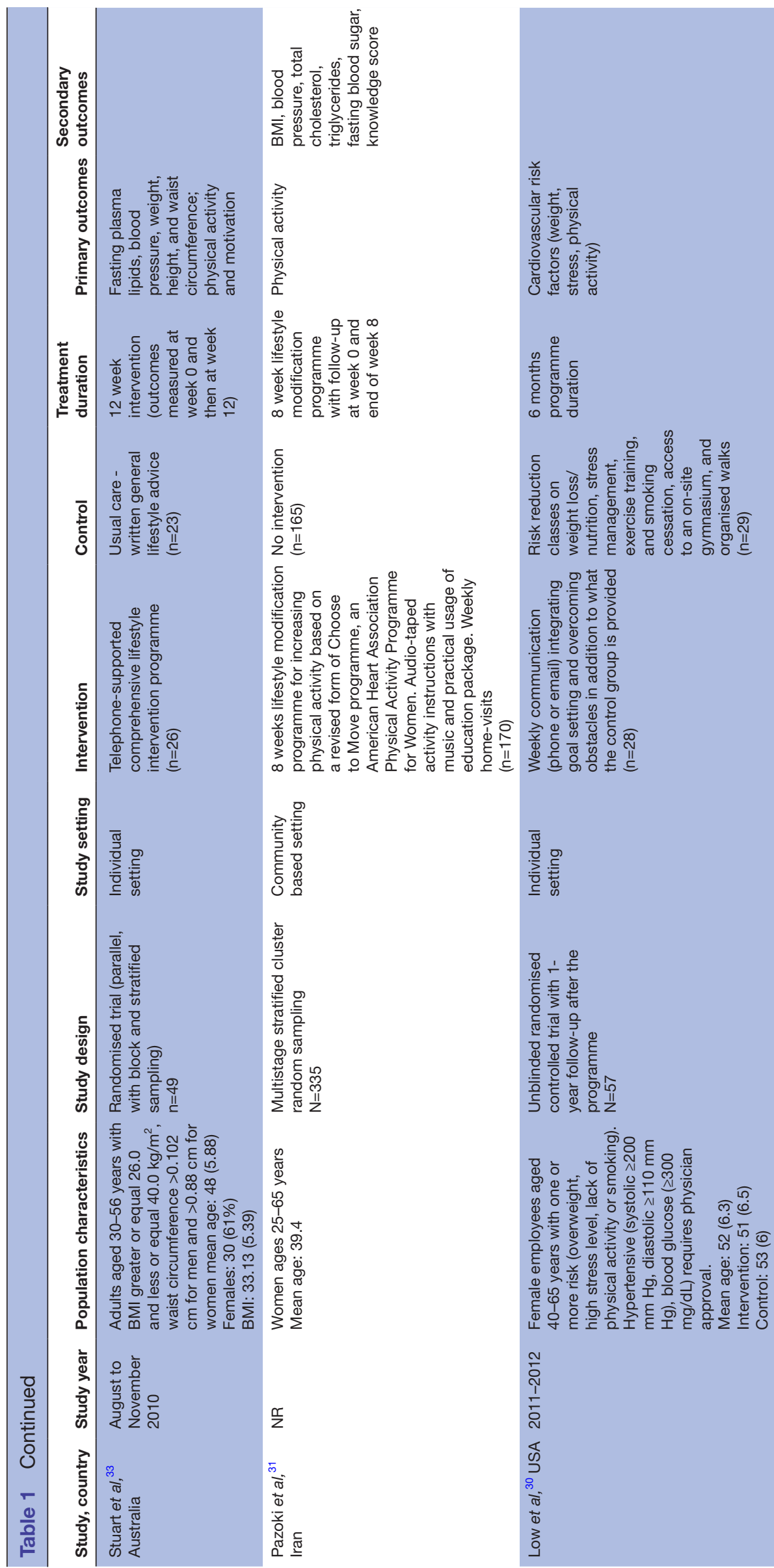

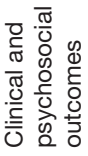

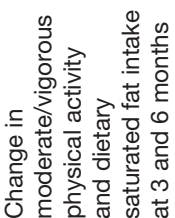

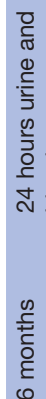

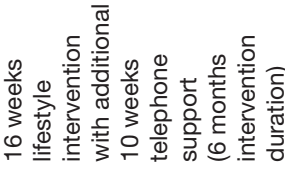
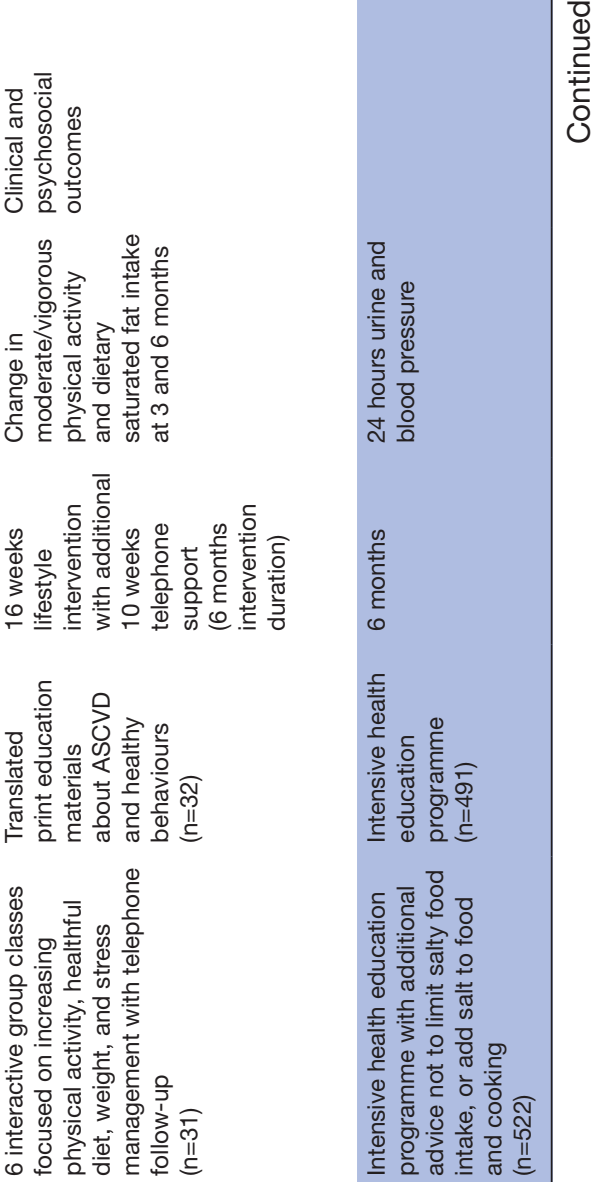

祍
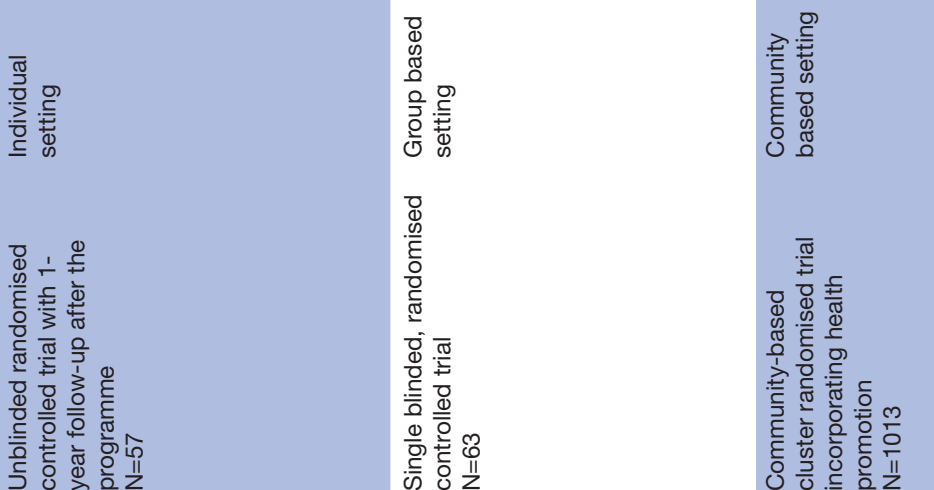

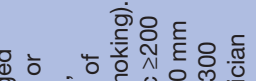

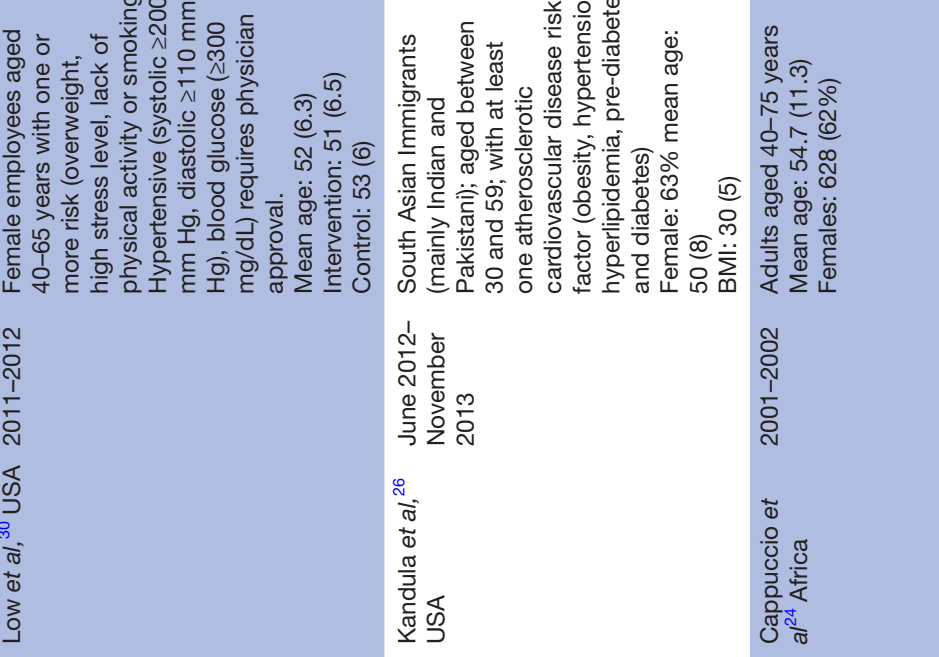




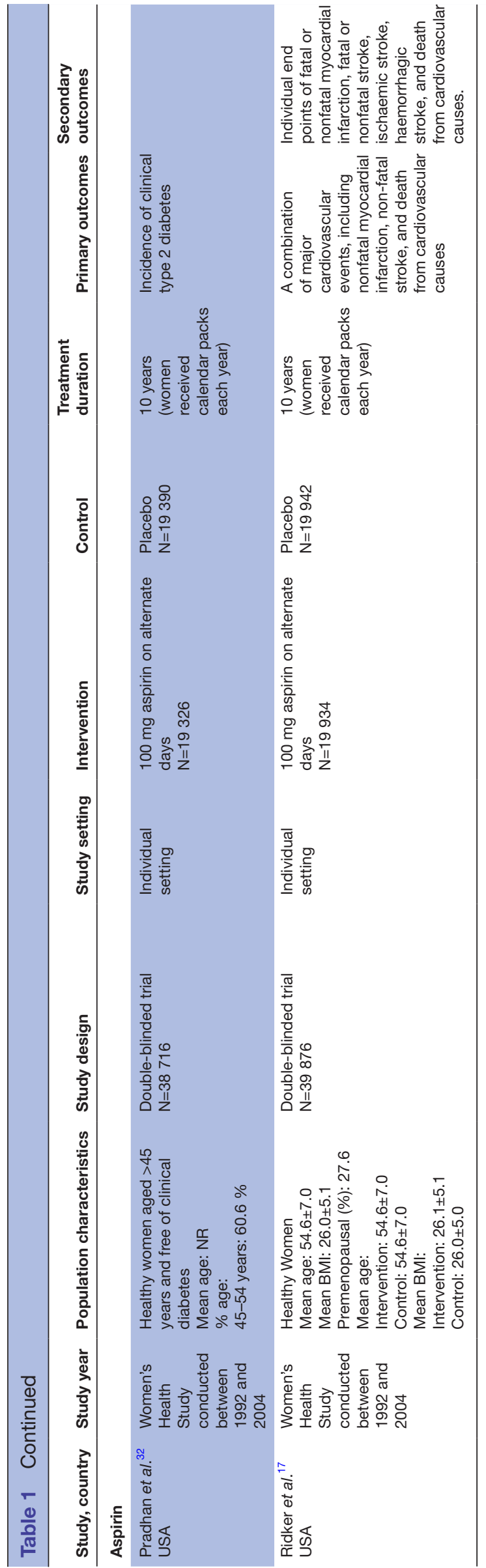

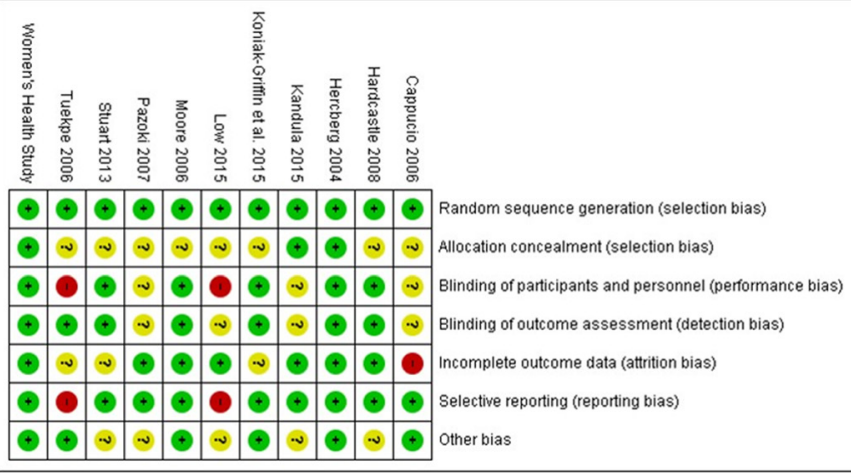

Green dot denotes low risk of bias; yellow dot denotes unclear risk of bias; red dot denotes high risk of bias

Figure 2 Risk of bias assessment of included studies.

three studies ${ }^{252735}$ with one or two domains of unclear risk; three studies ${ }^{26} 3133$ with three to four domains of unclear risk of bias; and three studies ${ }^{192}{ }^{24}$ with one or two high risk of bias domains rated. The detailed risk of bias assessment is provided in figure 2 .

\section{Results of quantitative synthesis}

Diet intervention

Two of the included studies related to dietary interventions. The study by Tuekpe et al trialled supplementing the diet of healthy women aged 18-38 years old with a combination of vegetables (ie, yellow-green Okinawan vegetables) over a 2 -week period. At the end of the intervention, they found a significantly increased bioavailability of urinary potassium (a mineral associated with reduced $\mathrm{BP}$ and heart attack), but no significant changes in lipid level. ${ }^{19}$

Another study by Moore et al tested the health benefits of the consumption of different types of fish (ie, white or oily) combined with replaced habitual household fats (sunflower or rapeseed oil) in an overweight mixed-sex population aged 35-60 years. ${ }^{35}$ The four interventional groups (two types of fish $\times$ two types of replaced oils) were meta-analysed synthetically and both studies included participants with a mean age under 51 years old. There was no significant difference in the change of lipid levels after this dietary intervention ( $p>0.05$ with all comparisons in triglycerides, cholesterol and low-density and high-density lipoproteins, $)$, body weight $(\mathrm{p}=0.93)$ and BP ( $>0.05$ for both systolic and diastolic), with no heterogeneity detected (all with $\mathrm{I}^{2}=0$ ). Forest plots showing results of the meta-analysis are available from online supplemental file 1 .

\section{Vitamin E/antioxidant}

Three articles from large trials (with 7713 participants in one study and more than 38000 subjects from the Women's Health study in the other two) investigated the efficacy of vitamin E in women exclusively. ${ }^{28294}$ The medium follow-up was more than 7 years in all three studies. Studies by Hercberg $e t a l$ and Lee $e t$ al reported the incidence (ie, de novo cases) of major cardiovascular events (ie, nonfatal MI, nonfatal stroke, or death from 
cardiovascular causes) and overall mortality over 10 years of follow-up, while Liu et al reported the incidence of type 2 diabetes. ${ }^{29}$ No meta-analysis was conducted due to the potential overlapping in the study populations recruited from the Women's Health study. RRs of reduction in incidence of major cardiovascular events, mortality and diabetes show no significant difference in each individual studies.

\section{Lifestyle modification intervention}

Seven studies focused on interventions involving a lifestyle modification programme in a community setting, which were grouped together for further analysis. ${ }^{24-27303133}$ Components of these interventions included physical activity, diet and lifestyle education/counselling, and multiple follow ups over time. Five $\mathrm{e}^{25-273033}$ out of seven studies focused on women with increased risk of CVD, while the other two recruited women without any CVD risk factors. ${ }^{24} 31$ The meta-analysis of six studies ${ }^{24-27} 3133$ suggested an overall reduction in the systolic BP by an MD) of $-2.11 \mathrm{~mm} \mathrm{Hg}\left(95 \% \mathrm{CI}-4.32\right.$ to $\left.0.11, \mathrm{p}=0.06, \mathrm{I}^{2}=0 \%\right)$ favouring the intervention group. The meta-analysis of five studies 24253133 showed a significant reduction also in diastolic $\mathrm{BP}$ with a reduction of $\mathrm{MD}$ of $-3.31 \mathrm{~mm} \mathrm{Hg}$ ( $95 \%$ CI -4.72 to $-1.91, \mathrm{p}<0.001, \mathrm{I}^{2}=0 \%$ ). The subgroup analysis by baseline risk of CVD suggested that for reduction in systolic BP, women without CVD risk factors $(-3.16$ $\mathrm{mm} \mathrm{Hg}, 95 \% \mathrm{CI}-6.32$ to $-0.01, \mathrm{p}=0.05, \mathrm{I}^{2}=0 \%$ ) tended to benefit more from the intervention than those with existing CVD risk factors $(-1.08 \mathrm{~mm} \mathrm{Hg}, 95 \%$ CI -4.19 to $2.03, \mathrm{p}=0.88, \mathrm{I}^{2}=0 \%$ ); on the other hand, for diastolic BP, both subgroups achieved similar significant reductions (figure 3). Among these studies, the meta-analysed

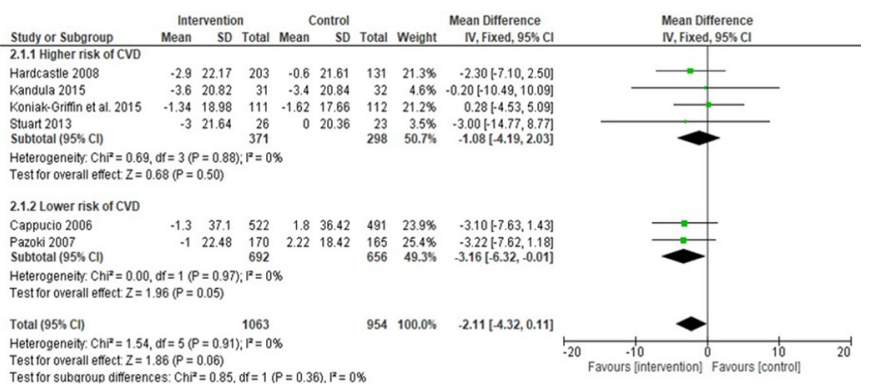

A

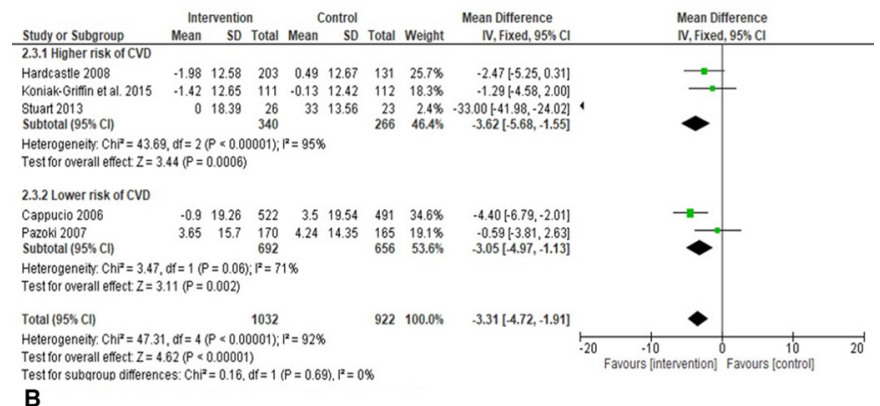

Figure 3 Forest plot of lifestyle modification intervention blood pressure. (A) Systolic blood pressure $(\mathrm{mmHg})$. (B) Diastolic blood pressure $(\mathrm{mmHg})$. CVD, cardiovascular disease.

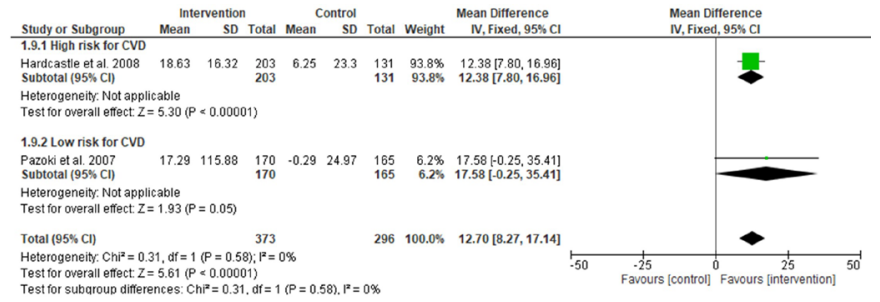

A

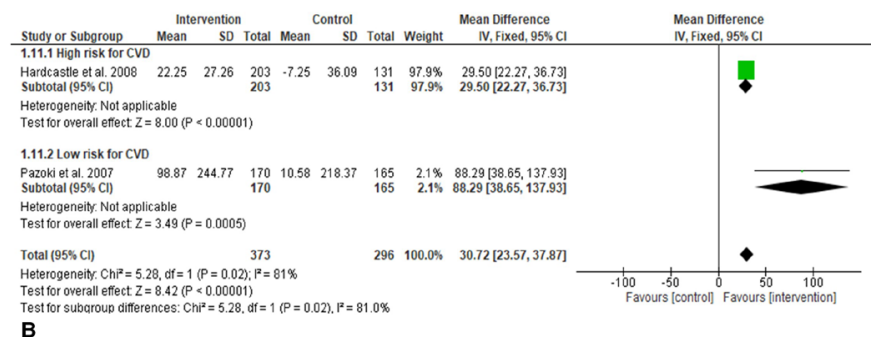

Figure 4 Forest plot of lifestyle modification intervention physical activity. (A) Vigorous physical activity (MET-min/ week). (B) Moderate physical activity (MET-min/week). CVD, cardiovascular disease.

results $(\mathrm{N}=669)^{25} 31$ also showed that there was a significant improvement in moderate and vigorous physical activity levels. MD of 30.72 Metabolic equivalent of task (MET)-min/week (95\% CI 23.57 to $37.87, \mathrm{p}=<0.001$, $\left.\mathrm{I}^{2}=81 \%\right)$ was reported for moderate physical activity and an MD of 12.70 MET-min/week (95\% CI 8.27 to 17.14 , $\mathrm{p}=<0.001, \mathrm{I}^{2}=0 \%$ ) for vigorous physical activity (figure 4 ). In addition, the meta-analysis ${ }^{262731}$ also indicated that the intervention led to significant reduction in fasting blood sugar $\left(-0.37 \mathrm{mmol} / \mathrm{L}, \mathrm{I}^{2}=76.4 \%, \mathrm{p}<0.001\right)$.

Subgroup analysis indicated that this intervention in lowering the systolic BP became insignificant by adopting a younger cut-off age for menopausal status (ie, $<51$ years instead of 55 years) while the improvements in physical activity and fasting blood glucose sustained. Further, women without baseline CVD risk factors were likely to experience greater reduction in fasting blood glucose (online supplemental figures).

\section{Aspirin}

Two publications ${ }^{17} 32$ each reported different outcomesincidence of major CVD events in one publication and incidence of type 2 diabetes in women treated with aspirin for a median of 10 years in the other-from the same long-term study (ie, Women's Health Study) which had a large sample size $(\mathrm{N}>37$ 000). Aspirin was shown to lower the occurrence of ischaemic stroke but did not alter the risk of MI or overall mortality over a 10-year follow-up; meanwhile, low dose of aspirin did not prevent the development of type 2 diabetes in healthy women as well. ${ }^{172}$ For the subgroup with premenopausal age (aged between 45 and 54 years), none of the studies ( $\mathrm{N}=24$ 025 in the CVD prevention and $\mathrm{N}=23473$ in the type 2 diabetes prevention studies, respectively) detected a significant intervention effect for the primary outcomes 
(ie, incidences of major CVD event, $\mathrm{p}=0.92$, and cases of type 2 diabetes, $\mathrm{p}=0.29$ ).

The detailed results of meta-analysis by intervention type are presented in online supplemental figures.

\section{DISCUSSION}

From 14 RCTs reviewed, it suggested that diet nor vitamin E/antioxidant did not significantly lower the CVD risk profiles, while lifestyle modification programme showed great potential to improve risk profiles via meta-analysing studies with smaller sample size and shorter duration of follow-up. The commonly used CVD prediction score illustrated the significant difference in coronary heart disease (CHD) risk between women and men before age of 70. It is reported that the onset of CHD in women lags behind that in men by $10-15$ years. ${ }^{36} 37$ This is echoed by our identified study that showed that aspirin was able to lower the risk of ischaemic stroke but not MI in women of mixed age (ie, women with mean age of 55). ${ }^{17}$ Recent data further suggests stagnation in the improvements in incidence and mortality of CHD, specifically among younger women ( $<55$ years). ${ }^{38}$ It is imperative that actions to be taken to reduce future CVD morbidity and mortality in young women with traditionally (ie, high BP and levels of lipids) and non-traditionally recognised risk factors that are unique to or more common in women (ie, histories of pregnancy-related complications or autoimmune diseases). ${ }^{39}$ Identifying interventions that could be effective for this age and gender group is essential given that CVD shows evident age and gender differences (ie, primary prevention interventions in older mixed-gender populations are not necessarily as effective in younger sex-specific populations). ${ }^{40}$ To fill this knowledge gap, our systematic review identified primary prevention interventions of CVD that had been tested in young women (ie, sage of 55) to fill; it will facilitate the design of clinical studies to examine the effectiveness of primary prevention for CVD in this population. The results from the systematic review and meta-analyses showed that diet or vitamin E/antioxidants supplement did not significantly improve CVD risk factors or alter the incidence of CVD; meanwhile, lifestyle modification programmes (incorporating a component of physical activity) had a moderate impact in terms of improving CVD risk factors.

A systematic review by Guirguis-Blake et al summarised evidence on the efficacy of aspirin for the primary prevention of CVD; it concluded that aspirin reduced the risk of nonfatal MI (RR $0.78,95 \%$ CI 0.71 to 0.87 ) but not nonfatal stroke in non-sex specific population, while older adults achieved greater relative MI reduction. ${ }^{42}$ One of the studies included in Guiguis-Blake systematic review, which was also included in our current review, showed a statistically significant $34 \%$ reduction in total MI only among women aged over 65 years (RR 0.66, $95 \%$ CI 0.44 to 0.97$).{ }^{17}$ Consistent with these findings, guidelines for the use of aspirin for CVD prevention take this gender difference into consideration: aspirin use in males is primarily intended for the prevention of CHD, while in females, prevention of stroke is the main target. ${ }^{43}$ Although the mechanism accounting for this gender difference is undisclosed, limited evidence suggests that there may be some biological basis for these differences (eg, baseline platelet reactivity is greater in women than in men, with higher residual reactivity following aspirin treatment in women). ${ }^{44}$ All this highlights the importance of considering sex together with the age difference in choosing an appropriate primary prevention of CVD in young women.

Contemporary clinical trials in the field of CVD often face the limitation of under-representation of female participants. ${ }^{45}$ Considering the well-recognised sexdifference in interventions targeting CVD, it is important to ascertain the intervention impact for women exclusively. To the best of our knowledge, there is no systematic review conducted to examine the efficacy of primary prevention interventions for CVD in premenopausal women (ie, age younger than 55 years). Existing reviews of primary prevention interventions of CVD in mixed age groups of women reported inconsistent results, and have primarily focused on pharmacological interventions. Trials focused on the efficacy of statins have generally recruited women of older age, and showed mixed effectiveness results. The JUPITER 2008 trial reported rosuvastatin significantly lowered the incidence of major cardiovascular events, ${ }^{13}$ while another meta-analysis synthesising evidence from six trials concluded that statins did not significantly impact on total or CHD mortality or major cardiovascular events. ${ }^{47}$ The most recent 2019 American College of Cardiology/AHA Guidelines on the primary prevention of CVD stipulates that weak evidence exists to support the use of statins in people with borderline CVD risk ( $<7.5 \%$ 10-year CVD risk) ${ }^{48}{ }^{49}$ Similarly, there is no strong evidence to support the administration of low-dose aspirin (75-100 mg/day) among adults 40-70 years old. ${ }^{48}$ However, these recommendations again highlight the importance of lifestyle factors that affect GVD risk.

Interventions that have a lifestyle modification component show a great potential for primary prevention of CVD in young women. The seven studies reviewed comprised various components that included increasing physical activity and/or healthy lifestyle through audiotaped activity instructions, written advice, with or without telephone/home visit/face-to-face counselling/follow-up. ${ }^{24-27} 303133$ Generally, these short-term studies (ie, follow-up ranged from 8 weeks to 6 months) found that the these interventions were able to improve some of the CVD risk factors (ie, physical activity time, BP, fasting blood glucose, etc). If a more comprehensive/effective lifestyle intervention is to be developed for young women, the components from each of the study could be extracted and combined. For example, daily activity instructions could be potentially united with face-to-face counselling or intensive health education programme to achieve a better outcome, by taking 
women's preferences and the advance in mobile technologies into consideration.

This is the first study to systematically review existing evidence on the primary prevention of CVD in women with reproductive age. Our systematic review did not include primary prevention intervention of CVD through the use of statins or hormone replacement therapy since these trials recruited women post-menopausal exclusively. This has significant importance since women are associated with sex-specific CVD risk factors that are acquired early in their life, for example, pregnancy-associated complications, oral contraceptives, polycystic ovary syndrome, autoimmune diseases, etc. However, in reality, if women do not have traditionally recognised CVD risk factors, they are not cared for by the usual primary prevention interventions targeted at CVD internationally. Early intervention for those at risk could prevent or postpone the onset of CVD disease later in the life. The potentially effective and ineffective interventions targeting primary prevention of CVD identified in this systematic review will provide fundamental evidence to guide the design of interventions and clinical trials.

Some limitations are worth mentioning. First, we were not able to obtain individual level data from included studies. The majority of studies only recruited a proportion of women aged younger than 55 years (ie, a crude proxy for the menopausal status) and did not report outcomes by age groups, with only one study focused on women aged 18-38 years. ${ }^{19}$ However, it is believed that evidence from studies incorporated premenopausal women could still form the basis for intervention selection targeted for this population. Particularly, for lifestyle modification interventions, three studies enrolled women exclusively and in the other four, women accounted for the majority of participants. The 1-year follow-up results from the study by Hardcastle et al reported that the benefits (eg, reductions inBP, weight and $\mathrm{BMI}$ ) observed during the 6 -month of intervention period did not sustain to 1 year. ${ }^{50}$ The decay of intervention effect raises a major concern from an implementation perspective. Further, especially for the studies investigated the lifestyle intervention, the difference in study design and intervention duration may impact on the conclusions drawn from them. Future clinical trials that are adequately powered and have longerterm follow-up, are warranted to investigate the long-term interventional impact in this population.

\section{CONCLUSION}

From this systematic review of primary prevention interventions of CVD in premenopausal women, it was concluded that vitamin E (based on large and relatively long-term follow-up RCTs) and diet interventions were not effective in lowering the CVD risk factors whereas lifestyle modification programmes involving modification of diet and/or physical activity (drew on small-sized and short-term follow-up RCTs) were effective in improving a series of biomarkers including diastolic BP, moderate to vigorous physical activity, and fasting blood glucose. Future primary prevention interventions for CVD in premenopausal women could be designed based on the effective interventions identified from this review.

Contributors LG and MM conceptualised and designed the review. JF, IM and PN reviewed titles, abstracts and full-text papers for inclusion. JF and IM independently assessed the quality of each included study. LG prepared the first draft of manuscript. LG, JF, IM, PN and MM reviewed and edited the manuscript, and approved the submission.

Funding LG is supported by the Alfred Deakin Postdoctoral Research Fellowship provided by the Deakin University, Australia.

Competing interests None declared.

Patient consent for publication Not required.

Provenance and peer review Not commissioned; externally peer reviewed.

Data availability statement Data sharing not applicable as no datasets generated and/or analysed for this study. Not applicable.

Supplemental material This content has been supplied by the author(s). It has not been vetted by BMJ Publishing Group Limited (BMJ) and may not have been peer-reviewed. Any opinions or recommendations discussed are solely those of the author(s) and are not endorsed by BMJ. BMJ disclaims all liability and responsibility arising from any reliance placed on the content. Where the content includes any translated material, BMJ does not warrant the accuracy and reliability of the translations (including but not limited to local regulations, clinical guidelines, terminology, drug names and drug dosages), and is not responsible for any error and/or omissions arising from translation and adaptation or otherwise.

Open access This is an open access article distributed in accordance with the Creative Commons Attribution Non Commercial (CC BY-NC 4.0) license, which permits others to distribute, remix, adapt, build upon this work non-commercially, and license their derivative works on different terms, provided the original work is properly cited, appropriate credit is given, any changes made indicated, and the use is non-commercial. See: http://creativecommons.org/licenses/by-nc/4.0/.

\section{ORCID iDs}

Lan Gao http://orcid.org/0000-0001-9734-1140

Phuong Nguyen http://orcid.org/0000-0001-7669-8642

\section{REFERENCES}

1 Lee SK, Khambhati J, Varghese T, et al. Comprehensive primary prevention of cardiovascular disease in women. Clin Cardiol 2017;40:832-8.

2 Kessous R, Shoham-Vardi I, Pariente G, et al. An association between preterm delivery and long-term maternal cardiovascular morbidity. Am J Obstet Gynecol 2013;209:368.e1-368.e8.

3 Veltman-Verhulst SM, van Rijn BB, Westerveld HE, et al. Polycystic ovary syndrome and early-onset preeclampsia: reproductive manifestations of increased cardiovascular risk. Menopause 2010;17:990-6.

4 Ray JG, Vermeulen MJ, Schull MJ, et al. Cardiovascular health after maternal placental syndromes (CHAMPS): population-based retrospective cohort study. Lancet 2005;366:1797-803.

5 Kruit MC, van Buchem MA, Hofman PAM, et al. Migraine as a risk factor for subclinical brain lesions. JAMA 2004;291:427-34.

6 Mancia G, Rosei EA, Ambrosioni E, et al. Hypertension and migraine comorbidity: prevalence and risk of cerebrovascular events: evidence from a large, multicenter, cross-sectional survey in Italy (miracles study). J Hypertens 2011;29:309-18.

7 Mosca L, Appel LJ, Benjamin EJ, et al. Evidence-based guidelines for cardiovascular disease prevention in women. American heart association scientific statement. Arterioscler Thromb Vasc Biol 2004;24:e29-50.

8 Bushnell C, McCullough LD, Awad IA, et al. Guidelines for the prevention of stroke in women: a statement for healthcare professionals from the American heart Association/American stroke association. Stroke 2014;45:1545-88.

9 Alpérovitch A, Kurth T, Bertrand M, et al. Primary prevention with lipid lowering drugs and long term risk of vascular events in older people: population based cohort study. BMJ 2015;350:h2335.

10 Guasch-Ferré M, Bulló M, Babio N. Mediterranean diet and risk of hyperuricemia in elderly subjects at high cardiovascular risk. Ann Nutr Metab 2013;62:17. 
11 Newman AB, Dodson JA, Church TS, et al. Cardiovascular events in a physical activity intervention compared with a successful aging intervention: the life study randomized trial. JAMA Cardiol 2016;1:568-74.

12 Liao JK. Rosuvastatin to prevent vascular events in men and women with elevated C-reactive protein. Curr Atheroscler Rep 2009;11:243-4.

13 Ridker PM, Danielson E, Fonseca FAH, et al. Rosuvastatin to prevent vascular events in men and women with elevated C-reactive protein. N Engl J Med 2008;359:2195-207.

14 Rossouw JE, Anderson GL, Prentice RL, et al. Risks and benefits of estrogen plus progestin in healthy postmenopausal women: principal results from the women's health Initiative randomized controlled trial. JAMA 2002;288:321-33.

15 Hsia J, Langer RD, Manson JE, et al. Conjugated equine estrogens and coronary heart disease: the women's health Initiative. Arch Intern Med 2006;166:357-65.

16 Montgomery PR, Berger LG, Mitenko PA, et al. Salicylate metabolism: effects of age and sex in adults. Clin Pharmacol Ther 1986;39:571-6.

17 Ridker PM, Cook NR, Lee I-M, et al. A randomized trial of low-dose aspirin in the primary prevention of cardiovascular disease in women. N Engl J Med 2005;352:1293-304.

18 Chae CU, Albert CM, Moorthy MV, et al. Vitamin E supplementation and the risk of heart failure in women. Circ Heart Fail 2012;5:176-82.

19 Tuekpe MK-N, Todoriki H, Sasaki S, et al. Potassium excretion in healthy Japanese women was increased by a dietary intervention utilizing home-parcel delivery of Okinawan vegetables. Hypertens Res 2006;29:389-96.

20 Faubion SS, Kuhle CL, Shuster LT, et al. Long-Term health consequences of premature or early menopause and considerations for management. Climacteric 2015;18:483-91.

21 Takahashi TA, Johnson KM. Menopause. Medical Clinics of North America 2015;99:521-34.

22 Nelson HD. Menopause. Lancet 2008;371:760-70.

23 The Cochrane Collaboration. Cochrance Handbook for systematic reviews of interventions version 5.1.0, 2011. Available: https:// handbook-5-1.cochrane.org/chapter_8/8_assessing_risk_of_bias_in_ included studies.htm

24 Cappuccio FP, Kerry SM, Micah FB, et al. A community programme to reduce salt intake and blood pressure in Ghana [ISRCTN88789643]. BMC Public Health 2006;6:13.

25 Hardcastle S, Taylor A, Bailey M, et al. A randomised controlled trial on the effectiveness of a primary health care based counselling intervention on physical activity, diet and CHD risk factors. Patient Educ Couns 2008;70:31-9.

26 Kandula NR, Dave S, De Chavez PJ, et al. Translating a heart disease lifestyle intervention into the community: the South Asian heart lifestyle intervention (SAHELI) study; a randomized control trial. BMC Public Health 2015;15:1064.

27 Koniak-Griffin D, Brecht M-L, Takayanagi S, et al. A community health worker-led lifestyle behavior intervention for Latina (Hispanic) women: feasibility and outcomes of a randomized controlled trial. Int J Nurs Stud 2015;52:75-87.

28 Lee I-M, Cook NR, Gaziano JM, et al. Vitamin E in the primary prevention of cardiovascular disease and cancer: the women's health study: a randomized controlled trial. JAMA 2005;294:56-65.

29 Liu S, Lee I-M, Song Y, et al. Vitamin E and risk of type 2 diabetes in the women's health study randomized controlled trial. Diabetes 2006;55:2856-62.

30 Low V, Gebhart B, Reich C. Effects of a worksite program to improve the cardiovascular health of female health care workers. $J$ Cardiopulm Rehabil Prev 2015;35:342-7.

31 Pazoki R, Nabipour I, Seyednezami N, et al. Effects of a communitybased healthy heart program on increasing healthy women's physical activity: a randomized controlled trial guided by community-based participatory research (CBPR). BMC Public Health 2007;7:216.
32 Pradhan AD, Cook NR, Manson JE, et al. A randomized trial of lowdose aspirin in the prevention of clinical type 2 diabetes in women. Diabetes Care 2009;32:3-8.

33 Stuart KL, Wyld B, Bastiaans K, et al. A telephone-supported cardiovascular lifestyle programme (clip) for lipid reduction and weight loss in general practice patients: a randomised controlled pilot trial. Public Health Nutr 2014;17:640-7.

34 Hercberg S, Galan P, Preziosi P, et al. The SU.VI.MAX study: a randomized, placebo-controlled trial of the health effects of antioxidant vitamins and minerals. Arch Intern Med 2004;164:2335-42.

35 Moore CS, Bryant SP, Mishra GD, et al. Oily fish reduces plasma triacylglycerols: a primary prevention study in overweight men and women. Nutrition 2006;22:1012-24.

36 Grundy SM, Balady GJ, Criqui MH, et al. Primary prevention of coronary heart disease: guidance from Framingham: a statement for healthcare professionals from the AHA Task force on risk reduction. American heart association. Circulation 1998;97:1876-87.

37 McSweeney JC, Rosenfeld AG, Abel WM, et al. Preventing and experiencing ischemic heart disease as a woman: state of the science: a scientific statement from the American heart association. Circulation 2016;133:1302-31.

38 Wilmot KA, O'Flaherty M, Capewell S, et al. Coronary heart disease mortality declines in the United States from 1979 through 2011: evidence for Stagnation in young adults, especially women. Circulation 2015;132:997-1002.

39 Garcia M, Mulvagh SL, Merz CNB, et al. Cardiovascular disease in women: clinical perspectives. Circ Res 2016;118:1273-93.

40 Cangemi R, Romiti GF, Campolongo G, et al. Gender related differences in treatment and response to statins in primary and secondary cardiovascular prevention: the never-ending debate. Pharmacol Res 2017;117:148-55.

41 Appelman $\mathrm{Y}$, van Rijn BB, Ten Haaf ME, et al. Sex differences in cardiovascular risk factors and disease prevention. Atherosclerosis 2015;241:211-8.

42 Guirguis-Blake JM, Evans CV, Senger CA, et al. Aspirin for the primary prevention of cardiovascular events: a systematic evidence review for the U.S. preventive services Task force. Ann Intern Med 2016;164:804-13.

43 US Preventive Services Task Force. Aspirin for the prevention of cardiovascular disease: U.S. preventive services Task force recommendation statement. Ann Intern Med 2009;150:396-404.

44 Qayyum R, Becker DM, Yanek LR, et al. Platelet inhibition by aspirin 81 and $325 \mathrm{mg} /$ day in men versus women without clinically apparent cardiovascular disease. Am J Cardiol 2008;101:1359-63.

45 Melloni C, Berger JS, Wang TY, et al. Representation of women in randomized clinical trials of cardiovascular disease prevention. Circ Cardiovasc Qual Outcomes 2010;3:135-42.

46 Scott PE, Unger EF, Jenkins MR, et al. Participation of women in clinical trials supporting FDA approval of cardiovascular drugs. J Am Coll Cardiol 2018;71:1960-9.

47 Walsh JME, Pignone M. Drug treatment of hyperlipidemia in women. JAMA 2004;291:2243-52.

48 Arnett DK, Blumenthal RS, Albert MA, et al. 2019 ACC/AHA guideline on the primary prevention of cardiovascular disease: a report of the American College of Cardiology/American heart association Task force on clinical practice guidelines. Circulation 2019;140.

49 Arnett DK, Blumenthal RS, Albert MA, et al. ACC/AHA guideline on the primary prevention of cardiovascular disease. Circulation 2019;2019.

50 Hardcastle SJ, Taylor AH, Bailey MP, et al. Effectiveness of a motivational interviewing intervention on weight loss, physical activity and cardiovascular disease risk factors: a randomised controlled trial with a 12-month post-intervention follow-up. Int J Behav Nutr Phys Act 2013;10:40. 